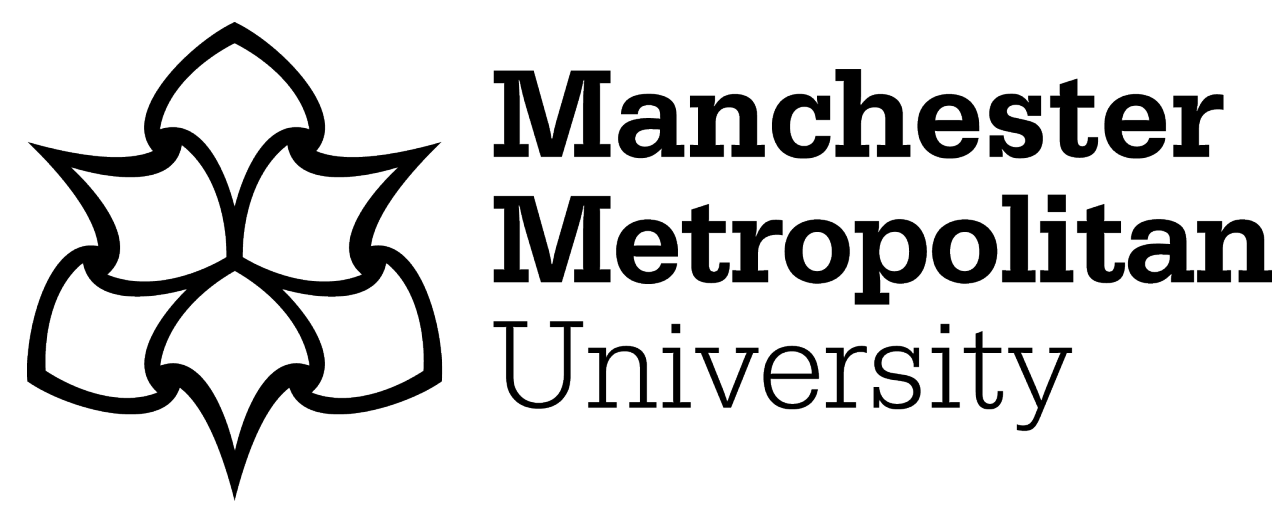

Tavassoli, A, Lim, C ORCID logoORCID: https://orcid.org/0000-0003-29168221, Kolodziej, J, Lauritzen, M, Knights, S, Wang, GG and Kjeang, E (2016) Effect of catalyst layer defects on local membrane degradation in polymer electrolyte fuel cells. Journal of Power Sources, 322. pp. 17-25. ISSN 03787753

Downloaded from: https://e-space.mmu.ac.uk/625204/

Version: Accepted Version

Publisher: Elsevier

DOI: https://doi.org/10.1016/j.jpowsour.2016.05.016

Please cite the published version 


\title{
Effect of catalyst layer defects on local membrane degradation in polymer electrolyte fuel cells
}

\author{
Arash Tavassoli a, Chan Lim ' , Joanna Kolodziej ${ }^{\text {b }}$, Michael Lauritzen ${ }^{\mathrm{b}}$, Shanna Knights ${ }^{\mathrm{b}}$, \\ G. Gary Wang a , Erik Kjeang ${ }^{\text {a, * }}$
}

${ }^{a}$ Fuel Cell Research Laboratory (FCReL), Simon Fraser University, 250-13450 102 Avenue, Surrey, BC, V3T 0A3, Canada

b Ballard Power Systems, 9000 Glenlyon Parkway, Burnaby, BC, V5J 5J8, Canada

\section{H I G H L I G H T S}

- Customized MEAs with artificial defects are subjected to accelerated stress tests.

- Significant global membrane thinning is observed in iron oxide contaminated MEAs.

- Negligible impact is shown for catalyst layer cracks and cathode delamination.

- Locally elevated membrane degradation is revealed at anode delamination sites.

- Substantial mitigating effect is shown for Pt residuals on the membrane surface.

\section{A R T I C L E I N F O}

Article history:

Received 19 February 2016

Received in revised form

4 May 2016

Accepted 5 May 2016

Keywords:

Fuel cell

Membrane degradation

Catalyst layer

Delamination

Crack

Iron contamination
G R A P H I C A L A B S T R A C T

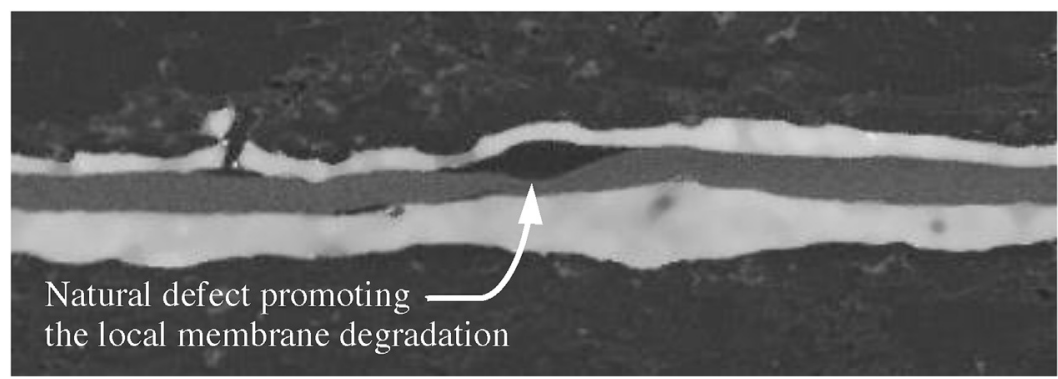

A B S T R A C T

Aiming at durability issues of fuel cells, this research is dedicated to a novel experimental approach in the analysis of local membrane degradation phenomena in polymer electrolyte fuel cells, shedding light on the potential effects of manufacturing imperfections on this process. With a comprehensive review on historical failure analysis data from field operated fuel cells, local sources of iron oxide contaminants, catalyst layer cracks, and catalyst layer delamination are considered as potential candidates for initiating or accelerating the local membrane degradation phenomena. Customized membrane electrode assemblies with artificial defects are designed, fabricated, and subjected to membrane accelerated stress tests followed by extensive post-mortem analysis. The results reveal a significant accelerating effect of iron oxide contamination on the global chemical degradation of the membrane, but dismiss local traces of iron oxide as a potential stressor for local membrane degradation. Anode and cathode catalyst layer cracks are observed to have negligible impact on the membrane degradation phenomena. Notably however, distinct evidence is found that anode catalyst layer delamination can accelerate local membrane thinning, while cathode delamination has no apparent effect. Moreover, a substantial mitigating effect for platinum residuals on the site of delamination is observed.

(c) 2016 Elsevier B.V. All rights reserved.

\footnotetext{
* Corresponding author.

E-mail address: ekjeang@sfu.ca (E. Kjeang).
}

\section{Introduction}

High durability and reliability are of increasing importance as 
fuel cell systems are widely deployed. Based on current market requirements, a lifetime of more than 20,000 and 6000 operating hours is targeted for buses and cars, respectively, while 40,000 h of operation is demanded for stationary applications [1]. Although the lifetime targets of automotive applications are considerably lower than those of stationary applications, the fluctuating operating conditions due to vehicle dynamics, variations in loads and environmental conditions, startups and shutdowns, fuel starvation, contamination, and freeze-thaw cycles pose a significant technological durability challenge. It has been demonstrated that different degradation routes can lead to failure of various cell components, but considering the expected service times of the fuel cells, the membrane is considered one of the most vulnerable components which predominantly determines the lifetime of a fuel cell stack. This has led to a large number of publications on known or hypothesized mechanisms for membrane degradation, under various working conditions and from different points of view [2].

The membrane degradation mechanisms that occur in polymer electrolyte fuel cells are not yet entirely known, but the progressive deterioration of membrane properties roots in a complex series of interconnected chemical and mechanical mechanisms. Chemical degradation is generally accepted as the key factor in the membrane degradation process where membrane stability is severely affected by the existence of reactive chemical species, i.e., radicals $[1,3]$. It has been confirmed via in-situ and ex-situ studies that the formation of free radicals during fuel cell operation plays a dominant role in the chemical degradation of the ionomer molecular structure in the membrane [4-8]; however, there are conflicting views on the formation and attack sites of these radicals. Huang et al. [9] and De Moor et al. [10] showed that degradation begins at the anode side and progresses toward the cathode, while others [11-13] have observed signs of predominant degradation on the cathode side. There are also evidences that degradation is uniform across the membrane thickness with no noticeable difference on either side [14]. Radicals can be produced through two distinct pathways [15], with $\mathrm{H}_{2} \mathrm{O}_{2}$ as the intermediate species or via direct reaction of $\mathrm{H}_{2}$ and $\mathrm{O}_{2}$ on the surface of $\mathrm{Pt}$ [16-21]. $\mathrm{H}_{2} \mathrm{O}_{2}$ would be formed on the anode or cathode catalyst layers. As proposed by Damjanovic et al. in the 1960s [22], oxygen is reduced via a direct four-electron oxygen reduction reaction (ORR), forming water. However, ORR may also proceed through two-electron reduction to $\mathrm{H}_{2} \mathrm{O}_{2}\left(E^{0}=0.695 \mathrm{~V}\right.$ vs. SHE) followed by the reduction of $\mathrm{H}_{2} \mathrm{O}_{2}$ to water $\left(E^{o}=1.77 \mathrm{~V}\right.$ vs. SHE). In this case, $\mathrm{H}_{2} \mathrm{O}_{2}$ may detach from the catalyst surface, enter the bulk of the membrane, and decompose to radicals in the presence of transition metal cations or heat $[4,11,23]$. Although the four-electron pathway is dominant on noble metals such as Pt, two-electron reduction occurs simultaneously in varying ratios [24]. It has also been suggested that the presence of carbon supported Pt catalyst [25] or Pt band in the membrane [26] originating from catalyst layer degradation may facilitate the kinetics for the formation of hydrogen peroxide under specific operating conditions. Finally, considering the reaction potentials, the anode side is a more favorable site for the two-electron reduction where the potential is lower than the reaction potential ( $0.695 \mathrm{~V}$ vs. SHE) and oxygen may be present due to gas crossover $[27,28]$.

Corrosion of stack components and external impurities originating from reactant air or humidifier reservoirs could bring small quantities of metal contaminants into the cells. Traces of metal impurities may also be detected in as-received membranes and other component materials. With emphasis on transportation applications in urban areas, fuel cell systems are also exposed to various air pollutants present in the environment. After extended operation, the membrane electrode assembly (MEA) of the fuel cell is expected to contain measurable amounts of $\mathrm{Ca}^{2+}, \mathrm{Fe}^{3+}, \mathrm{Cu}^{2+}$, $\mathrm{Na}^{+}, \mathrm{K}^{+}$, and $\mathrm{Mg}^{2+}[1]$. The presence of impurities such as $\mathrm{Fe}^{3+}$ ions can contribute to the chemical membrane degradation process by catalyzing the radical formation reactions [29]. In a recent work by Wong et al. [30], a transient membrane degradation model was developed to capture the redox cycle of iron ions within the membrane electrode assembly, which sustains the presence of harmful $\mathrm{Fe}^{2+}$ ions in the membrane even at low contamination levels and results in the most severe chemical degradation at open circuit voltage. As originally proposed by LaConti et al. [31], hydroxyl (HO-) and hydroperoxyl (HOO-) radicals are formed from hydrogen peroxide in the presence of these ions.

Commonly used perfluorosulfonic acid (PFSA) ionomer membranes are composed of a PTFE backbone featuring periodic fluorous-ether side chains with sulfonic acid end groups, providing mechanical stability and proton conductivity, respectively [32,33]. It was first determined that chemical degradation is predominantly initiated through $\mathrm{OH}$ radical attack on the main chain carboxylic acid terminal groups $[16,34-36]$ or on the $\mathrm{C}-\mathrm{S}$ or $\mathrm{O}-\mathrm{C}$ bonds of the side chain [15,16,35-42]. However, as new generations of membranes have been chemically stabilized, the concentration of terminal carboxylic acid groups has been decreased to negligible levels $[34,35]$ and degradation therefore occurs mainly on the side chain of the ionomer [43].

While chemical degradation mechanisms are responsible for the gradual deterioration of membrane properties, mechanical degradation is considered as a major cause for sudden membrane failures. Originating from hygrothermal cycles during fuel cell operation, varying temperature and relative humidity result in repetitive expansion and contraction of the membrane, inducing fatigue, creep, and tension which ultimately leads to cracks and holes associated with membrane failure [44-52]. Manufacturing imperfections can introduce local stress points where cracks and holes may evolve [53-55], while certain operating conditions such as low humidity $[20,55,56]$ can render the membrane brittle and fragile, making it more vulnerable to further mechanical stress.

Although the majority of available literature focuses on membrane degradation as a global phenomenon, it is generally accepted that catastrophic membrane failures are due to local failure points [57] leading to destructive reactant gas transfer between the opposing electrodes. It is therefore hypothesized that local defects and variations in the MEA structure may influence the local rates of membrane degradation and form preferential sites for eventual failure. Kundu et al. [53] categorized the possible morphological defects imparted on catalyst coated membranes in their beginningof-life state. It is assumed that these defects are mainly introduced during the manufacturing and processing. They classified these anomalies into six major categories of catalyst layer cracks, delamination, orientation, electrolyte clusters, platinum clusters, and thickness variations, among which the first two are expected to be of primary relevance for membrane degradation. Cracks are prone to form in fresh and aged MEAs and are commonly present in both anode and cathode catalyst layers. A fuel cell can generally operate in the presence of such defects; however, performance and durability may be negatively affected. For a fresh MEA, these features could be formed during manufacturing or be a consequence of poor handling, bending, or stretching of the MEA. Membrane expansion and contraction during fuel cell operation may also result in catalyst layer cracks [45,51,53]. Delamination, which is observed when the catalyst layer is separated from the membrane, may also occur during the manufacturing processes or during operation [45,51]. As discussed by Kundu et al. [53], the high temperature applied in the catalyst layer drying stage can cause vapor to form at the catalyst layer/membrane interface, and create areas of poor adhesion. Differences in thermal and expansion properties of membranes and catalyst layers may also be responsible for delamination during both manufacturing and operation. It 
is postulated that such local imperfections may function as local stress points and therefore contribute to local membrane degradation and failure over time.

The main objective of the present work is to determine potential correlations between pre-existing catalyst layer related defects and local membrane degradation. With a novel experimental approach, an in-situ screening experiment is conducted with the aim of elucidating possible causes of local membrane degradation, local thinning, and pinhole formation as initiators to membrane failure. Based on a comprehensive review of historical membrane failure analysis data from field operation, local sources of iron oxide contaminants, catalyst layer cracks, and catalyst layer delamination are selected as the main defects of interest. In order to determine their effect on membrane degradation, customized MEAs with artificial defects are designed and fabricated, and subjected to accelerated stress testing followed by extensive post-mortem characterization and analysis.

\section{Experimental}

\subsection{Membrane electrode assembly (MEA) fabrication}

Catalyzed gas diffusion electrodes (GDEs) were fabricated by coating a micro-porous layer made of polytetrafluoroethylene (PTFE) and carbon black on a non-woven carbon paper gas diffusion layer substrate, followed by coating a catalyst layer consisting of carbon-supported platinum catalyst and perfluorosulfonic acid ionomer [58]. Regular MEAs were prepared by hot-pressing a standard perfluorosulfonic acid ionomer membrane with anode and cathode GDEs. Customized MEAs containing artificial defects were prepared as follows.

\subsubsection{Local sources of iron oxide contamination}

Iron (III) oxide $\mathrm{Fe}_{2} \mathrm{O}_{3}$ particles were locally deposited at the membrane/cathode catalyst layer interface before hot-pressing of the GDEs and membrane. Iron (III) oxide powder was supplied by Sigma-Aldrich with particle size of $<5 \mu \mathrm{m}$. Drops of $2 \mu \mathrm{l}$ aqueous solution containing Fe concentrations of 100, 200, and 1000 ppm (mg Fe per $1 \mathrm{~kg}$ of membrane) were deposited on the membrane surface at periodic locations, each covering a circular area of $2 \mathrm{~mm}$ in diameter. The solution was air dried on the membrane surface at ambient temperature before assembly and hot-pressing. Fig. 1(a) shows a cross-sectional scanning electron microscope (SEM) image of a locally contaminated region in the obtained MEA.

\subsubsection{Local catalyst layer cracks}

Customized MEAs were fabricated with local regions of missing catalyst layer, simulating enlarged versions of catalyst layer cracks.
Using standard GDEs, the catalyst layer was deliberately scraped off the electrode in circular areas of $1-2.5 \mathrm{~mm}$ in diameter at periodic locations on either anode or cathode GDEs. The obtained GDEs were assembled into MEAs using the previously described procedure. Fig. 1(b) shows a cross-sectional SEM image of a customized MEA with simulated cathode catalyst layer cracks.

\subsubsection{Local catalyst layer delamination}

Delamination was simulated by inserting a $9 \mu \mathrm{m}$ thick porous, hydrophilic film at periodic locations between the membrane and anode/cathode catalyst layers (CLs). The film was supplied by Sterlitech Corp. and is a Polycarbonate Track Etch (PCTE) PVPcoated membrane made from micro porous polycarbonate film material with nominal pore size of $0.8 \mu \mathrm{m}$ and pore density of $3 \cdot 10^{7}$ pores $\mathrm{cm}^{-2}$. It has good resistance to acidic environments and can withstand operating temperatures up to $140{ }^{\circ} \mathrm{C}$. Two cases of membrane/CL delamination were simulated: i) delamination with bare membrane; and ii) delamination with catalyst particles remaining on the membrane surface. In the latter case, considering the potential impact of catalyst residuals on the chemical degradation process, supplementary analysis was performed with small amounts of catalyst ink sprayed on one side of the film. 32 circular pieces (1-2.5 $\mathrm{mm}$ in diameter) of film (with and without catalyst spray) were positioned between the membrane and either the anode or cathode CL and assembled into MEAs. Fig. 1(d) shows the schematic configuration of artificial delamination while Fig. 1(c) shows a cross-sectional SEM image of an obtained MEA with cathode delamination along with remainders of catalyst ink on the membrane side of the film located at the membrane/CL interface.

\subsection{Stack assembly}

Four customized MEAs, simulating local sources of iron oxide contamination, local anode and cathode catalyst layer cracks, local anode delamination, and local cathode delamination, and one regular, defect-free baseline MEA were assembled into a researchscale five-cell stack. The cells were separated by graphitic bipolar plates, where a pressurized bladder ensured uniform compression between MEAs and plates during operation. The flow field plates had co-flow parallel straight channels and high gas flow rate conditions, ensuring minimum pressure drop and minimum changes in reactant composition from stack inlet to outlet, resulting in quasi-uniform operating conditions. Prior to installation on a test station, external and internal gas leak tests were carried out by pressurizing dead-ended fluid compartments and measuring any gas transfer to other compartments.
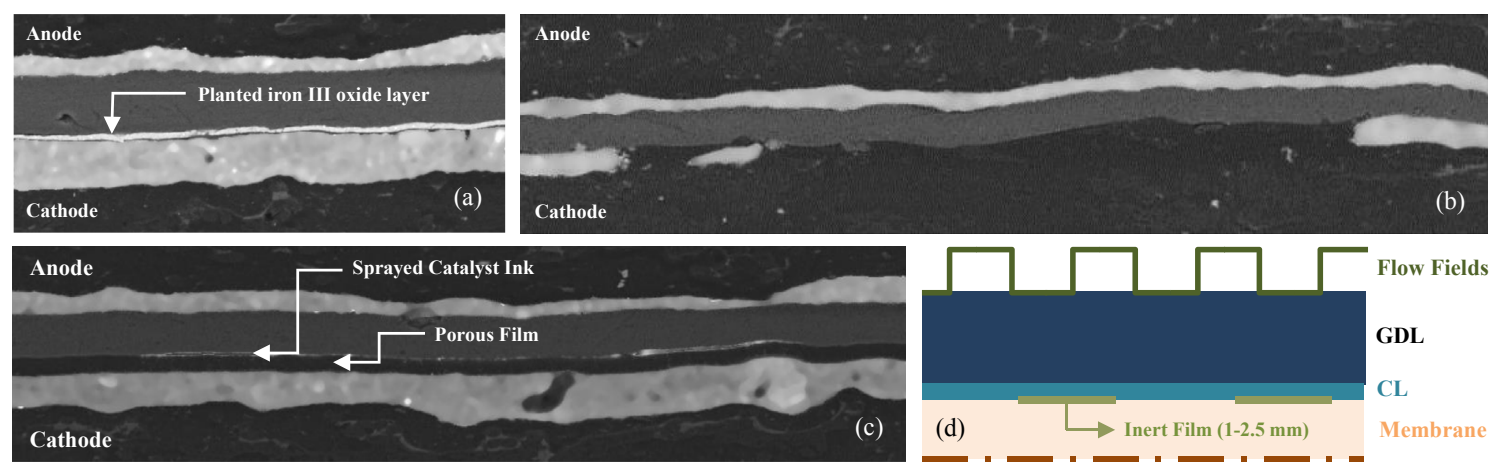

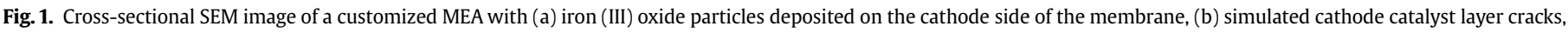

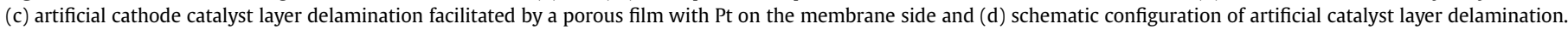




\subsection{Accelerated stress test protocols}

Designed to evaluate combined chemical and mechanical membrane durability, Ballard Power Systems' cyclic open-circuit voltage (COCV) accelerated stress test (AST) [59] protocol was used to expose the membranes to in-situ chemical and mechanical stresses. The COCV-AST applies chemical and mechanical stress in an alternating pattern, which is repeated until membrane failure is obtained. A COCV-AST cycle features an open circuit voltage (OCV) phase under high temperature and low RH conditions, followed by a series of wet/dry cycles in pure nitrogen. During the OCV phase, the membrane is subjected to high chemical degradation due to high cell temperature, high potential, high oxygen partial pressure, and low humidity [59-62], while during the wet/dry cycles, the membrane is subjected to high mechanical stress due to repeated swelling in the wet-phase and shrinkage in the dry-phase [63]. The stack was conditioned before launch of the COCV-AST by applying a medium current density in pure hydrogen and air on anode and cathode, respectively.

While the COCV-AST is an aggressive test that is mainly intended for stability benchmarking of new materials, our group recently reported a more moderate accelerated stress test for membrane durability estimation of fuel cell stacks considering duty cycle and key stressors of field operated MEAs. The proposed accelerated membrane durability test (AMDT) [21,64] exposes the MEA to lower temperature and higher RH levels than the COCV-AST, with less aggressive wet-dry cycling. Also, instead of the OCV condition, the AMDT maintains the cell voltage at $0.9 \mathrm{~V}$. The lower temperature, besides the non-OCV condition, reduces the rate of chemical membrane degradation while the milder wet and dry phases reduce the level of mechanical membrane degradation. The AMDT protocol, which is more representative of field operation, was used in this work to validate the preliminary COCV-AST results.

The two tests were carried out on an automated test station, consisting of fully computer-controlled mass flow controllers (MFCs), back-pressure control system, pre-heated water circulating coolant loops, and electronic load. The test station was equipped with two water-injected evaporator type humidifiers capable of controlling RH levels instantaneously without long warm-up and cool-down preconditioning time for humidity control.

\subsection{Diagnostics and post-mortem analysis}

The health of the membranes was monitored periodically via an electrochemical leak detection test (ELDT) [59], where hydrogen transfer leaks were recorded by measuring the OCV before and after applying a small hydrogen overpressure in a pure hydrogen and air condition on anode and cathode, respectively. The OCV condition dictates negligible activation, ohmic, and concentration polarization, hence the amount of increase in hydrogen crossover is correlated to the OCV difference before and after applying the overpressure. The operating voltage and current of each cell were also monitored continuously and used as an indicator of performance degradation.

Scanning electron microscopy (SEM) was used to visualize the effect of local defects on membrane thinning and damage. For this purpose, cross-sectional SEM was the most advantageous approach in order to capture the internal features of the local MEA regions of interest. The MEAs were cut through the center of the regions of interest, cast in epoxy pucks, polished using a Struers TegraPol-11 polisher with 120-1200 grit silicon carbide paper, top-coated with carbon using an Edwards Scancoat Six Sputter Coater, and analyzed with a Philips XL30 scanning electron microscope.

\section{Results and discussion}

Customized MEAs, simulating local sources of iron oxide contaminants, catalyst layer cracks, and electrode delamination were fabricated and tested for membrane stability under COCV-AST and AMDT conditions. Hereafter, online monitoring and diagnostic records of the two stacks are reported, followed by post-mortem failure analysis of individual cells for the signs of degradation.

In order to accelerate chemical and mechanical membrane degradation, the COCV-AST applies combined chemical and mechanical stressors, resulting in significantly shorter lifetimes than for isolated chemical or mechanical tests [59]. The present five-cell stack was operated under COCV-AST conditions until failure was observed on all five cells, based on the two specific failure criteria of OCV dropping below $0.8 \mathrm{~V}$ or ELDT reaching $40 \mathrm{mV}$. This occurred after $142 \mathrm{~h}$, equal to nine AST cycles.

Fig. 2(a) shows the change in open circuit voltage associated with the five individual cells during the COCV-AST operation. Each cyclic section of this graph shows one AST cycle, including a constant OCV phase followed by wet/dry cycling. Different individual rates of performance decay were observed. The decrease in OCV mainly arose due to mixed electrode potentials resulting from increased hydrogen crossover through the degraded membranes and subsequent oxidation in the presence of oxygen on the cathode. In order to validate the results from COCV-AST, a second stack with similar configuration was prepared and tested under AMDT operating conditions, which are milder and more representative of field operation. Fig. 2(b) shows the voltage decay profile of the five individual cells during the AMDT operation. For safety reasons, the two cells with iron oxide contaminants and catalyst layer cracks were replaced after reaching the ELDT failure criterion at the end of the 6th AMDT cycle. The test was terminated upon failure of the baseline MEA as well as the cell with cathode delaminations, both based on high ELDT signals. The ELDT signal for the cell with anode delamination did not reach the failure criterion; however, the test was terminated after $412 \mathrm{~h}$, equal to eight AMDT cycles.

\subsection{Local sources of iron oxide contamination}

Under COCV-AST conditions, the cell with iron oxide contamination at the membrane/cathode interface was the first cell which failed, with its OCV reaching the failure criterion of $0.8 \mathrm{~V}$ at the end of the 7th COCV-AST cycle (107 h). Concurrently, the cell's ELDT signal also indicated failure at the end of the 7th cycle. Fig. 2(a) shows that for most of its operation time, the cell had lower OCV than the other cells, including the baseline. As expected, this was accompanied by considerable growth in hydrogen crossover measured by ELDT. A significant negative effect for iron contaminations on membrane lifetime was thus identified; however, after surveying the post-mortem MEA under the SEM, no correlation could be established between the initial positions of local sources of iron oxide and the points of failure. Fig. 3(a) shows a locally contaminated region with iron (III) oxide particles, while Fig. 3(b) and (c) show two random locations on the clean areas of the same MEA, both after $142 \mathrm{~h}$ of operation under COCV-AST conditions. As representatively illustrated in these images, the locations of severe membrane degradation were entirely random and there was no consistent correlation between the location of leak transfer points and the initial deposition locality of the contaminant.

The cell with iron oxide contamination was also the first cell that failed under AMDT conditions due to high hydrogen crossover measured by ELDT. Similar to the COCV-AST results, post-mortem characterization showed no signs of locally accelerated degradation in the regions where $\mathrm{Fe}_{2} \mathrm{O}_{3}$ was originally deposited, regardless of their concentration. Failure analysis of both cells found the leak 


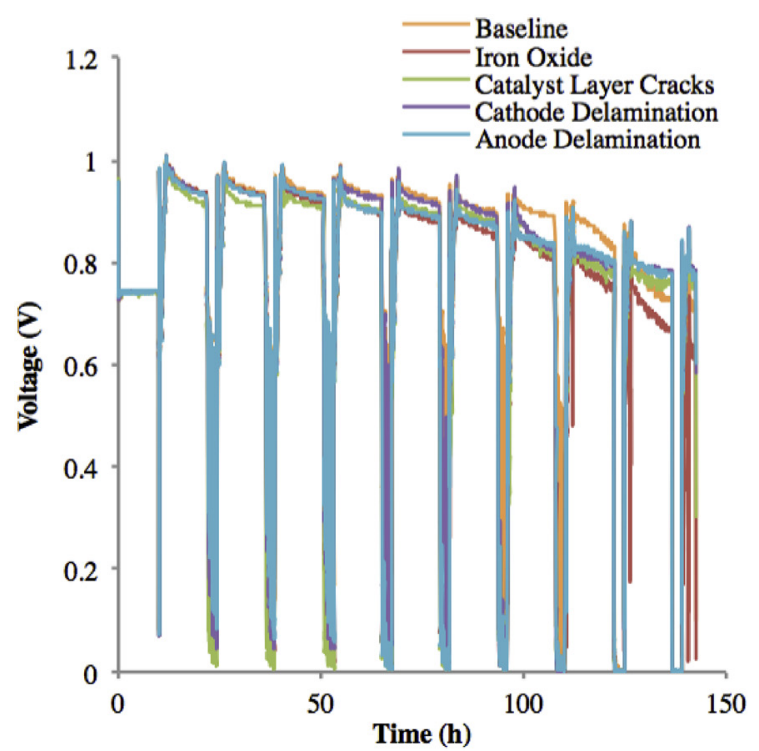

(a)

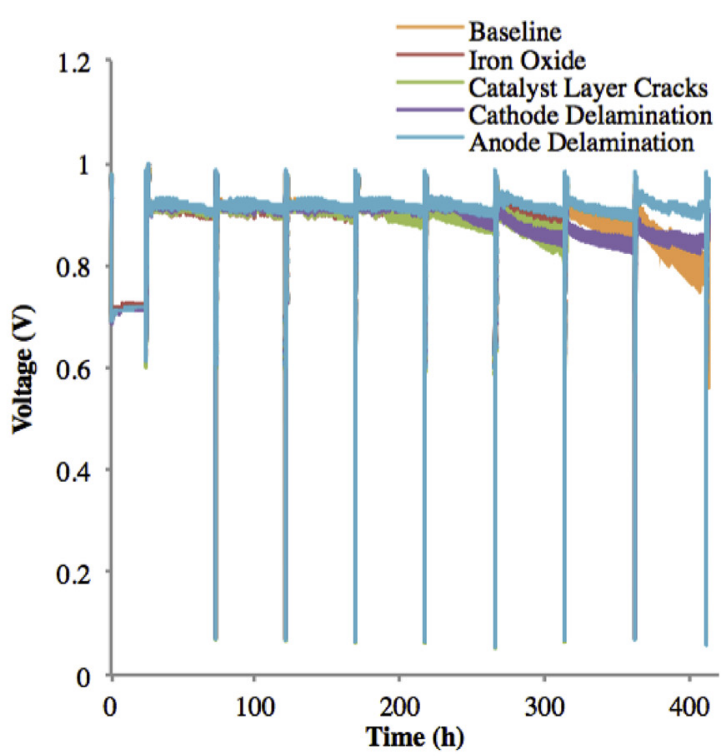

(b)

Fig. 2. Voltage profile of the five-cell stacks during (a) COCV-AST and (b) AMDT operation.

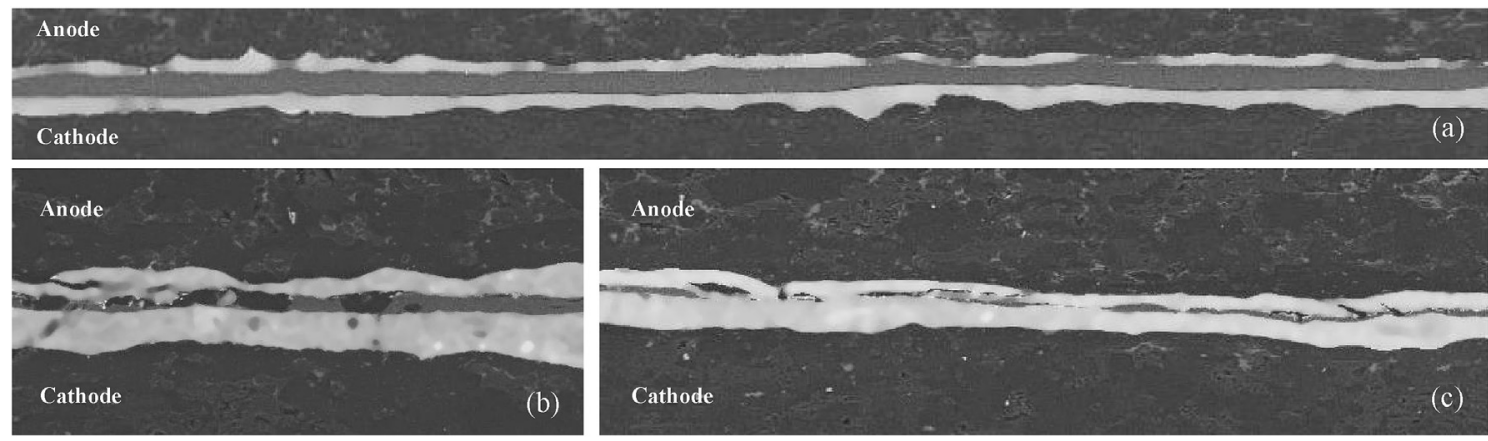

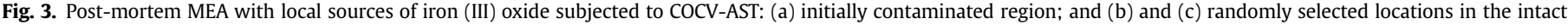
region of the same MEA.

transfer points to be randomly located across the active area of the MEAs, suggesting that the contaminants were uniformly distributed throughout the membrane at an early stage of the test, acting as a global rather than local accelerating factor. This is theoretically supported by the high solubility of $\mathrm{Fe}_{2} \mathrm{O}_{3}$ in acidic environments [65]. Surveying the post-mortem membrane surface, iron oxide particles could no longer be visualized, which confirms their complete dissolution during operation. This finding was consistent for a wide range of iron oxide concentrations of 100,200 , and $1000 \mathrm{ppm}$ applied in various regions of the MEAs. Assuming a uniform fully dissolved distribution of contaminants in the bulk of the membrane, the global iron concentration in the membrane was estimated to be $45 \mathrm{ppm}$ ( $45 \mathrm{mg}$ Fe per $1 \mathrm{~kg}$ of membrane).

\subsection{Local catalyst layer cracks}

Customized MEAs were fabricated to simulate cathode and anode catalyst layer cracks and tested under COCV-AST conditions. As shown in Fig. 2(a), the cell with artificial CL cracks showed a notable drop in OCV from the first cycle and failed during the 8th cycle. However, despite its early stage OCV drop, this cell showed a smooth decay in performance with a relatively low rate of hydrogen crossover. The local CL crack sites of the end of test (EOT) MEA were inspected using cross-sectional SEM, with selected images presented in Fig. 4. Notable discrepancies were observed at the crack sites compared to the intact regions. Considerable local variations in membrane thickness were found, but there was no sign of additional thinning or damage at the CL crack regions. Contrarily, the membrane even appeared to be thicker in several CL crack regions. Similar trends were observed for testing under AMDT conditions (not shown here for brevity).

Membrane thickness measurements on the COCV-AST and AMDT EOT MEAs, as shown in Fig. 5, coincided with the crosssectional SEM observations. Considering the relatively high variability, there was no statistically significant difference between the

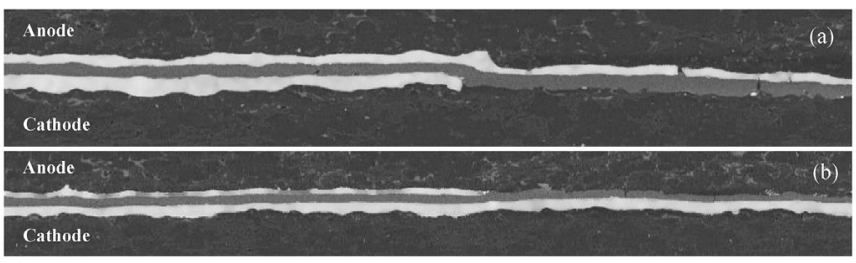

Fig. 4. Post-mortem MEA with local catalyst layer cracks on (a) cathode and (b) anode subjected to COCV-AST 
- Untouched

- Anode CL Cracks

- Cathode CL Cracks

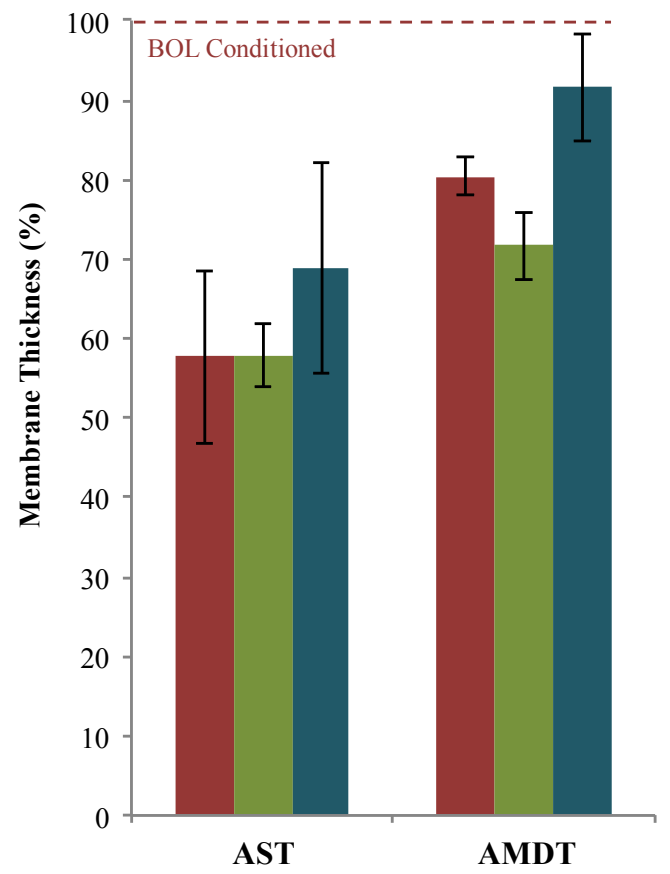

Fig. 5. End-of-test membrane thickness data for cells with artificial catalyst layer cracks tested under COCV-AST and AMDT conditions.

local membrane thinning in the regions with and without CL cracks on either electrodes for either of the two tests. Secondly, the large error bars depict the considerable inconsistencies in membrane thickness. This led to the conclusion that catalyst layer cracks, in the simulated size and shape, have no negative effect on membrane degradation. However, the cracks influenced the cell performance from the beginning of operation. Comparing the COCV-AST and AMDT online diagnostics, the cells with artificial cracks had a voltage profile slightly lower than the other cells for most of their operation period, which can be expected due to the relatively large size of the simulated cracks. This inferiority was retained until the other cells showed their first signs of mid-life degradation, where voltage losses due to hydrogen leaks overshadowed the initial voltage losses due to CL cracks. This finding was consistent for both COCV-AST and AMDT experiments, although less severe membrane degradation was observed under the milder AMDT conditions.

\subsection{Local catalyst layer delamination}

Two separate cells, each incorporating 32 artificially delaminated spots at the cathode and anode sides, respectively, were tested under COCV-AST conditions. The cell with artificial membrane/cathode CL delamination showed low rate of gas crossover given its low ELDT at EOT. In comparison, the cell with artificial membrane/anode CL delamination showed a higher rate of gas crossover for the entire operation time. As shown in Fig. 2(a), the cell with anode delamination also demonstrated lower OCV at midlife compared to the cell with cathode delamination. A significant contrast between the two cells was revealed by post-mortem SEM analysis. Fig. 6(a) represents a local region with artificial cathode delamination at EOT, indicating that the membrane thickness was comparable between the regions with and without artificial

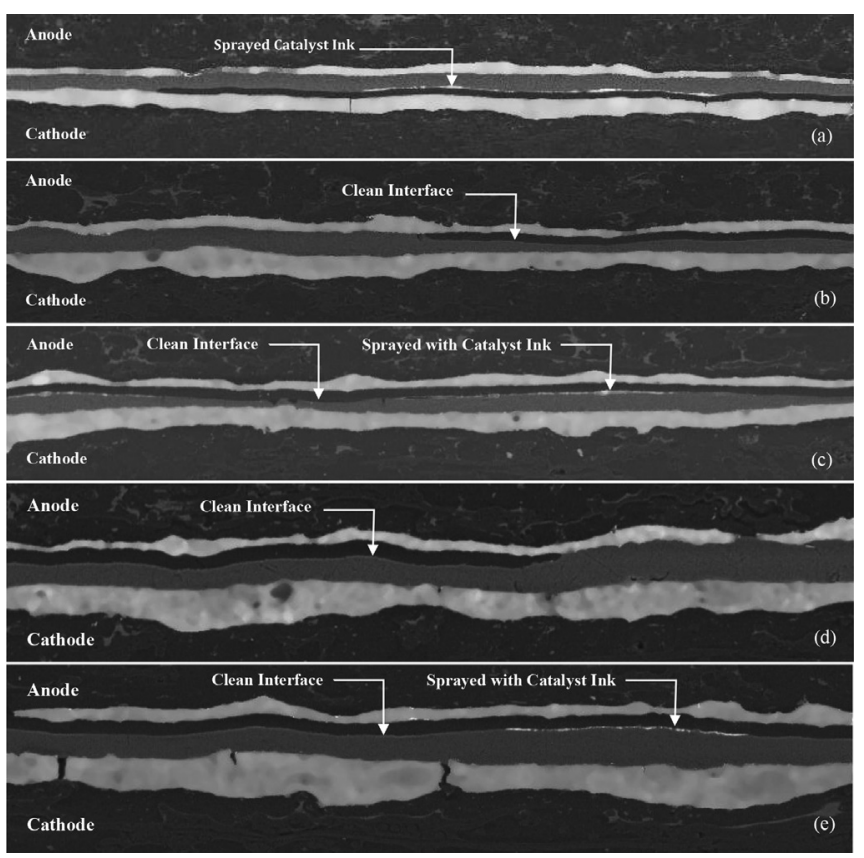

Fig. 6. Post-mortem MEA with (a) local cathode delamination subjected to COCV-AST, (b-c) local anode delamination subjected to COCV-AST with and without catalyst ink residuals, and (d-e) local anode delamination subjected to AMDT with and without catalyst ink residuals.

delamination. It is also shown that the presence of catalyst ink particles located at the membrane/cathode CL interface did not have an obvious accelerating effect on the degree of membrane thinning. Similar observations were made on the MEAs subjected to AMDT. Hence, it was concluded that cathode delamination had no significant accelerating effect on membrane thinning, and that the presence of platinum particles at the membrane/cathode $\mathrm{CL}$ interface had no major impact on the membrane stability under COCVAST and AMDT conditions.

One the other hand, Fig. 6(b) represents a local region with artificial anode delamination from the second cell tested under COCV-AST conditions. While both cells were tested under identical operating conditions for the same operation time, the regions with artificial anode delamination were considerably thinner at EOT, suggesting an accelerating effect for anode delamination on local membrane thinning.

In the case of anode delamination with catalyst residuals coated on the membrane surface, a considerable difference in thinning pattern was noted. Fig. 6(c) shows a delaminated spot on which small remainders of catalyst ink were present on the surface of the membrane. Overall, the membrane appeared to be thicker at the delaminated areas with catalyst residues than at the sites without catalyst residues. This observation is in agreement with a recently developed hypothesis published by Macauley et al. [21,64,66], where a mitigating effect for the Pt band inside the membrane was reported. It is known that Pt catalyzes $\mathrm{H}_{2} \mathrm{O}_{2}$ decomposition into harmless products of oxygen and water; hence, it is hypothesized that the catalyst remainders contributed to local hydrogen peroxide removal and thereby decreased the rate at which harmful radicals were formed. The thin Pt layer could also act as a barrier for crossover gases by converting them to water before they reach the opposing electrodes.

As shown in Fig. 6(d-e), the cell tested under AMDT conditions exhibited a similar trend. The membrane regions situated under the artificial anode delamination sites were observed to be thinner 
than the untouched regions of the same MEA at EOT. Also, sites with catalyst residuals at the membrane surface were observed to be thicker than similar delaminated regions where a clean film was used for mimicking the delamination.

Statistical measurements of membrane thickness at randomly selected spots along these MEAs are summarized in Fig. 7 for the purpose of verifying the aforementioned claims. At EOT, the membrane regions under the artificial anode delamination sites were observed to be thinner than those under intact sites. It is also shown that the membrane thickness at the delaminated sites was higher in the presence of catalyst residuals, indicating less severe thinning than in the case of pure delamination without catalyst residuals at the membrane surface. On the other hand, the membrane under the local cathode delamination sites appeared to have a comparable thickness to that of the intact regions, suggesting that the membrane thinning was independent of local cathode delamination under COCV-AST and AMDT conditions. An independentsample t-test was used to confirm the statistical significance of these findings. A significant difference between intact regions and areas with clean anode delamination was confirmed at $99 \%$ significance level in both cases of COCV-AST and AMDT. Moreover, a significant difference between anode delamination with and without $P$ t residuals was confirmed at $94 \%$ and $98 \%$ significance levels for COCV-AST and AMDT conditions, respectively.

Further measurements showed a considerable difference between the hydrophobicity of the membrane (with average static contact angle of $89.6^{\circ}$ ) and the inert polycarbonate film (with average static contact angle of $47.8^{\circ}$ ) used to create artificial delamination. The hydrophilic nature of the porous film and its affinity for liquid water may have affected the local transport mechanisms from the catalyst layer to the membrane. Considering the structural similarity of hydrogen peroxide and water, and the relatively low concentrations of this species in a working fuel cell, a comparable transport mechanism for $\mathrm{H}_{2} \mathrm{O}_{2}$ molecules may be assumed. Therefore, in a comparison between the intact regions and the local delamination sites, an enhanced hydrogen peroxide

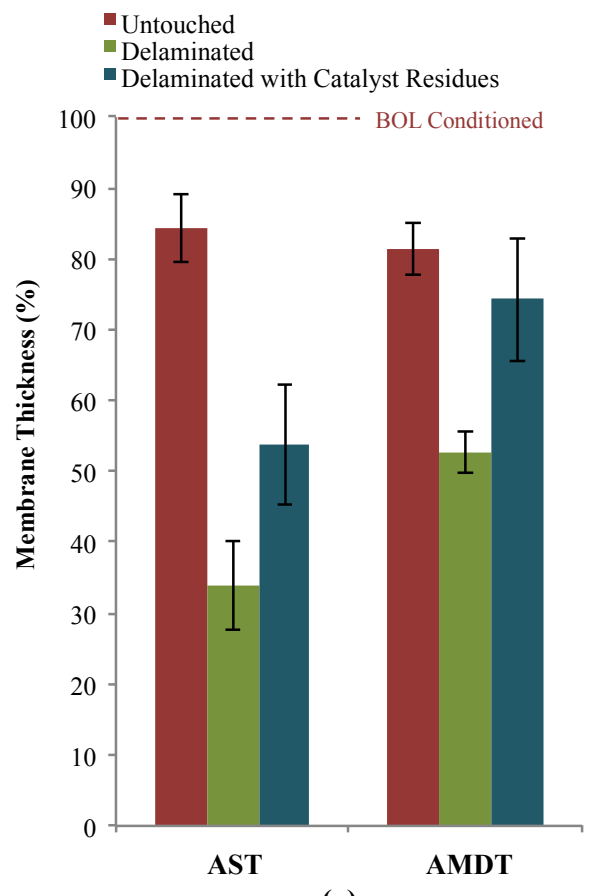

(a) transfer from the catalyst layer toward the membrane may occur in the presence of artificial delamination. As explained previously, electrochemical $\mathrm{H}_{2} \mathrm{O}_{2}$ generation via two-electron ORR is more favorable on the anode, especially in the case of OCV or near-OCV cell potentials $[28,30] . \mathrm{H}_{2} \mathrm{O}_{2}$ as a reaction product of the twoelectron ORR or intermediate species of the full, four-electron ORR can be detached from the catalyst surface before completing the reduction reaction and form radicals in the presence of Fenton's reagents in the membrane. Based on the present findings, it is hypothesized that the gap provided by the film at the delamination sites enhanced the hydrogen peroxide transfer from the anode catalyst layer toward the membrane, detaching $\mathrm{H}_{2} \mathrm{O}_{2}$ before further electrochemical reduction to water or chemical decomposition to water and oxygen, and thereby accelerated the local chemical degradation rate. In contrast, isolated platinum particles at the membrane surface are expected to provide local reaction sites for hydrogen peroxide decomposition to harmless water and oxygen, hence alleviating the rate of local chemical degradation. These effects are independent of cathode delamination provided the negligible rate of hydrogen peroxide formation on that electrode compared to the anode.

Finally, Fig. 8 shows a naturally evolved anode delamination site in an MEA operated under identical COCV-AST conditions for $142 \mathrm{~h}$. Enhanced local membrane thinning is observed at the site of anode delamination, in agreement with the local thinning trend observed

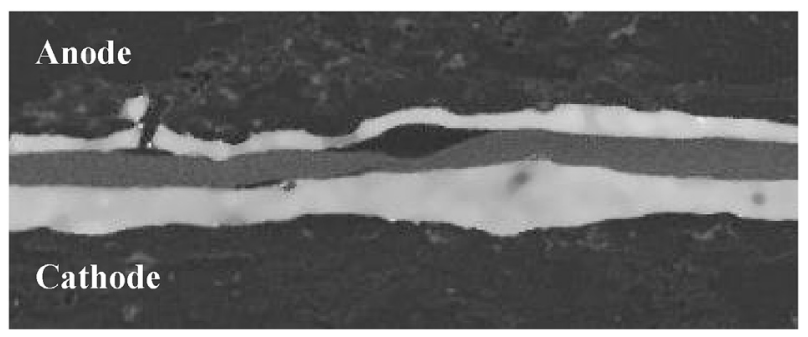

Fig. 8. Naturally evolved anode delamination observed after COCV-AST operation.

-Untouched

- Delaminated

- Delaminated with Catalyst Residues

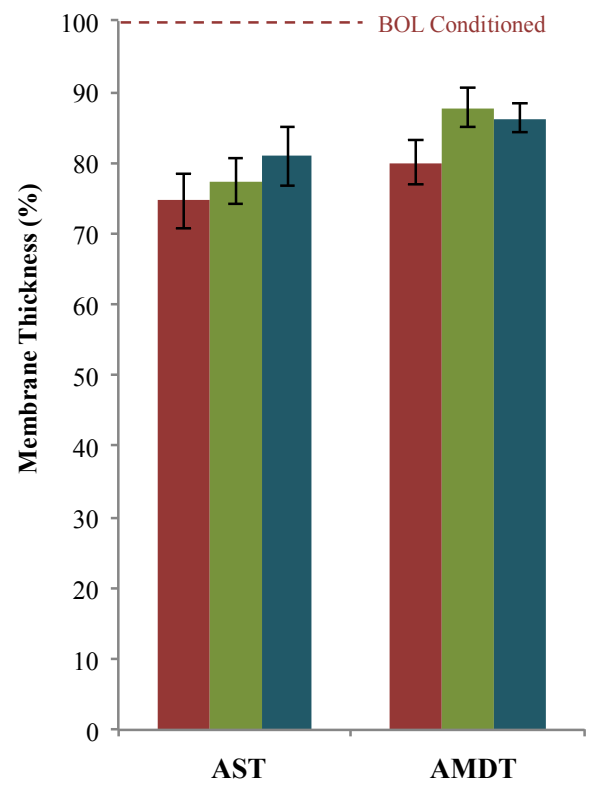

(b)

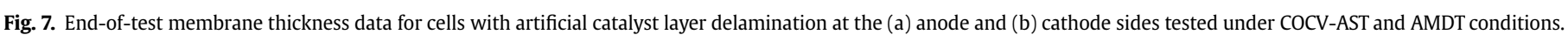


previously at the regions with artificially created anode delamination. This verifies the validity of the test design implemented in this work along with the results obtained.

\section{Conclusions}

Local membrane degradation was evaluated for automotive applications of polymer electrolyte fuel cells. With the aid of comprehensive failure analysis data from field operated MEAs, three alternatives were selected as potential candidates for initiating or accelerating local membrane degradation: local sources of iron oxide contaminants, catalyst layer cracks, and electrode delamination. Customized MEAs were designed and fabricated in order to artificially create these imperfections, and by subjecting these MEAs to accelerated stress tests followed by extensive failure analysis, this work aimed to establish a correlation between catalyst-layer related defects and local membrane degradation phenomena in fuel cells.

The diagnostic results of the contaminated cells with aqueous solution of iron (III) oxide confirmed a significant negative effect for iron contamination on membrane lifetime. Under two different test conditions, these cells failed earlier than the cells without applied contaminant. However, the failure analysis showed no meaningful correlation between location or concentration of contaminant source and the points of failure. While initially, different concentrations of iron oxide solution were deposited on traceable local spots, the end of test (EOT) MEAs showed a globally thinned membrane with randomly distributed points of failure. These observations strongly confirm an accelerating effect for iron contamination on chemical membrane degradation, but dismiss the local traces of iron oxide as local initiators or accelerators of this process.

With a comparable approach, it was shown that artificial anode and cathode cracks had no significant effect on membrane degradation phenomena under the timeframe and conditions of these accelerated stress and durability tests. Given longer term operation under less chemically stressful, more mechanically intense conditions, or with different electrode geometry at the crack site, different results may be obtained.

The potential effects of artificial anode and cathode catalyst layer delamination were evaluated under four different scenarios: local minuscule delamination with and without catalyst residue on the membrane surface, applied separately at anode and cathode locations. While cathode delamination appeared to have no significant effect on local membrane degradation, results from both accelerated stress and durability tests showed that the anodedelaminated sites experienced the harshest degrading environment with severe local thinning in both tests. In addition, the sites with catalyst residue on the membrane surface, simulating remainders of catalyst layer on the delaminated membrane, showed the capability to mitigate the locally accelerated membrane degradation. It is hypothesized that the local hydrophilic environment at the anode delamination sites facilitated express hydrogen peroxide release and transfer from the anode catalyst layer into the membrane, leading to increased rates of local chemical membrane degradation. Consequently, anode delamination events occurring during manufacturing or operation may cause preferential sites for local pinhole and crack development as an initiator to membrane failure, presumably under a wide range of conditions given the consistent results for both accelerated stress and durability tests. Further considering the high catalytic activity of Pt deposited on the membrane surface, the accelerating effect of anode delamination may be suppressed by the decomposition of incoming hydrogen peroxide into benign water and oxygen in lieu of radical formation in the membrane.
The present work focuses on degradation of non-reinforced perfluorosulfonic acid ionomer membranes. As future work, it would be valuable to apply a similar methodology to study the effects of catalyst layer imperfections on multilayer composite membranes, and to systematically evaluate and compare the degradation trends for different commercially available membranes.

\section{Acknowledgments}

Funding for this research provided by Automotive Partnership Canada (APC), Natural Sciences and Engineering Research Council of Canada (NSERC), and Ballard Power Systems is gratefully acknowledged. Ballard Power Systems is also acknowledged for providing access to experimental facilities and technical support. The authors thank Lida Ghassemzadeh for her persistent support and advice, and Anudeep Chohan, Ted Hung, and Ileana Co for assisting with experimentation and characterization.

\section{References}

[1] A. Collier, H. Wang, X. Ziyuan, J. Zhang, D. Wilkinson, Int. J. Hydrogen Energy 31 (2006) 1838

[2] R. Borup, J. Meyers, B. Pivovar, Y.S. Kim, R. Mukundan, N. Garland, D. Myers, M. Wilson, F. Garzon, D. Wood, P. Zelenay, K. More, K. Stroh, T. Zawodzinski, J. Boncella, J.E. McGrath, M. Inaba, K. Miyatake, M. Hori, K. Ota, Z. Ogumi, S. Miyata, A. Nishikata, Z. Siroma, Y. Uchimoto, K. Yasuda, K.-I. Kimijima, N. Iwashita, Chem. Rev. 107 (2007) 3904.

[3] F.A. de Bruijn, V.A.T. Dam, G.J.M. Janssen, Fuel Cells 8 (2008) 3.

[4] M. Danilczuk, F.D. Coms, S. Schlick, J. Phys. Chem. B 113 (2009) 8031.

[5] A. Panchenko, H. Dilger, J. Kerres, M. Hein, A. Ullrich, T. Kaz, E. Roduner, Phys Chem. Chem. Phys. 6 (2004) 2891.

[6] A. Bosnjakovic, S. Schlick, J. Phys. Chem. B 108 (2004) 4332.

[7] M.K. Kadirov, A. Bosnjakovic, S. Schlick, J. Phys. Chem. B 109 (2005) 7664.

[8] M. Danilczuk, A. Bosnjakovic, M.K. Kadirov, S. Schlick, J. Power Sources 172 (2007) 78.

[9] C. Huang, K. Seng Tan, J. Lin, K. Lee Tan, Chem. Phys. Lett. 371 (2003) 80.

[10] G. De Moor, C. Bas, N. Charvin, J. Dillet, G. Maranzana, O. Lottin, N. Caqué, E. Rossinot, L. Flandin, Int. J. Hydrogen Energy 41 (2016) 483.

[11] A. Pozio, R.F. Silva, M. De Francesco, L. Giorgi, Electrochim. Acta 48 (2003) 1543.

[12] S. Stucki, G.G. Scherer, S. Schlagowski, E. Fischer, J. Appl. Electrochem. 28 (1998) 1041.

[13] J. Yu, B. Yi, D. Xing, F. Liu, Z. Shao, Y. Fu, H. Zhang, Phys. Chem. Chem. Phys. 5 (2003) 611.

[14] B. Mattsson, H. Ericson, L. Torell, F. Sundholm, Electrochim. Acta 45 (2000) 1405.

[15] L. Ghassemzadeh, K.D. Kreuer, J. Maier, K. Müller, J. Power Sources 196 (2011) 2490.

[16] L. Ghassemzadeh, K.D. Kreuer, J. Maier, K. Müller, J. Phys. Chem. C 114 (2010) 14635.

[17] J. Xie, D.L. Wood, K.L. More, P. Atanassov, R.L. Borup, J. Electrochem. Soc. 152 (2005) A1011.

[18] V.O. Mittal, H.R. Kunz, J.M. Fenton, J. Electrochem. Soc. 153 (2006) A1755.

[19] V.O. Mittal, H.R. Kunz, J.M. Fenton, J. Electrochem. Soc. 154 (2007) B652.

[20] E. Endoh, S. Terazono, H. Widjaja, Y. Takimoto, Electrochem. Solid-State Lett. 7 (2004) A209.

[21] N. Macauley, K.H. Wong, M. Watson, E. Kjeang, J. Power Sources 299 (2015) 139.

[22] A. Damjanovic, J. Chem. Phys. 45 (1966) 4057

[23] T. Okada, H. Satou, M. Yuasa, Langmuir 19 (2003) 2325.

[24] A.A. Shah, T.R. Ralph, F.C. Walsh, J. Electrochem. Soc. 156 (2009) B465.

[25] W. Liu, D. Zuckerbrod, J. Electrochem. Soc. 152 (2005) A1165.

[26] A. Ohma, S. Yamamoto, K. Shinohara, J. Power Sources 182 (2008) 39.

[27] V.A. Sethuraman, J.W. Weidner, A.T. Haug, S. Motupally, L.V. Protsailo, J. Electrochem. Soc. 155 (2008) B50.

[28] K.H. Wong, E. Kjeang, J. Electrochem. Soc. 161 (2014) F823.

[29] M. Inaba, T. Kinumoto, M. Kiriake, R. Umebayashi, A. Tasaka, Z. Ogumi, Electrochim. Acta 51 (2006) 5746

[30] K.H. Wong, E. Kjeang, ChemSusChem 8 (2015) 1072

[31] A.B. LaConti, M. Mamdan, R.C. McDonald, Handbook of Fuel Cells vol. 3, John Wiley \& Sons, New York, 2003.

[32] K.A. Mauritz, R.B. Moore, Chem. Rev. 104 (2004) 4535.

[33] S.V. Venkatesan, C. Lim, E. Rogers, S. Holdcroft, E. Kjeang, Phys. Chem. Chem. Phys. 17 (2015) 13872.

[34] D.E. Curtin, R.D. Lousenberg, T.J. Henry, P.C. Tangeman, M.E. Tisack, J. Power Sources 131 (2004) 41.

[35] C. Zhou, M.A. Guerra, Z.-M. Qiu, T.A. Zawodzinski, D.A. Schiraldi, 
Macromolecules 40 (2007) 8695.

[36] F.D. Coms, in: ECS Trans., ECS, 2008, pp. 235-255.

[37] T.H. Yu, Y. Sha, W.-G. Liu, B. V Merinov, P. Shirvanian, W.A. Goddard, J. Am. Chem. Soc. 133 (2011) 19857.

[38] L. Ghassemzadeh, M. Marrony, R. Barrera, K.D. Kreuer, J. Maier, K. Müller, J. Power Sources 186 (2009) 334.

[39] T. Tokumasu, I. Ogawa, M. Koyama, T. Ishimoto, A. Miyamoto, in: ECS Trans., ECS, 2009, pp. 765-772.

40] M. Takasaki, Y. Nakagawa, Y. Sakiyama, K. Tanabe, K. Ookubo, N. Sato, T. Minamide, H. Nakayama, M. Hori, in: ECS Trans., ECS, 2009, pp. 439-447.

[41] W.E. Delaney, W. Liu, in: ECS Trans., ECS, 2007, pp. 1093-1104.

[42] A.M. Dreizler, E. Roduner, Fuel Cells 12 (2012) 132.

[43] L. Ghassemzadeh, S. Holdcroft, J. Am. Chem. Soc. 135 (2013) 8181.

[44] A. Sadeghi Alavijeh, M.A. Goulet, R.M.H. Khorasany, J. Ghataurah, C. Lim, M. Lauritzen, E. Kjeang, G.G. Wang, R.K.N.D. Rajapakse, Fuel Cells 15 (2015) 204.

[45] A. Sadeghi Alavijeh, R.M.H. Khorasany, A. Habisch, G.G. Wang, E. Kjeang, J. Power Sources 285 (2015) 16.

[46] R.M.H. Khorasany, M.A. Goulet, A.S. Alavijeh, E. Kjeang, G.G. Wang, R.K.N.D. Rajapakse, J. Power Sources 252 (2014) 176.

[47] R.M.H. Khorasany, A. Sadeghi Alavijeh, E. Kjeang, G.G. Wang, R.K.N.D. Rajapakse, J. Power Sources 274 (2015) 1208.

[48] R.M.H. Khorasany, E. Kjeang, G.G. Wang, R.K.N.D. Rajapakse, J. Power Sources 279 (2015) 55.

[49] M.A. Goulet, R.M.H. Khorasany, C. De Torres, M. Lauritzen, E. Kjeang, G.G. Wang, N. Rajapakse, J. Power Sources 234 (2013) 38

[50] M.A. Goulet, S. Arbour, M. Lauritzen, E. Kjeang, J. Power Sources 274 (2015) 94.

[51] A.S. Alavijeh, R.M.H. Khorasany, Z. Nunn, A. Habisch, M. Lauritzen, E. Rogers, G.G. Wang, E. Kjeang, J. Electrochem. Soc. 162 (2015) F1461.
[52] G. De Moor, N. Charvin, C. Bas, N. Caque, E. Rossinot, L. Flandin, IEEE Trans. Ind. Electron 62 (2015) 5275.

[53] S. Kundu, M.W. Fowler, L.C. Simon, S. Grot, J. Power Sources 157 (2006) 650.

[54] R. Lin, E. Gülzow, M. Schulze, K. a. Friedrich, J. Electrochem. Soc. 158 (2011) B11.

[55] J. Healy, C. Hayden, T. Xie, K. Olson, R. Waldo, M. Brundage, H. Gasteiger, J. Abbott, Fuel Cells 5 (2005) 302.

[56] S.D. Knights, K.M. Colbow, J. St-Pierre, D.P. Wilkinson, J. Power Sources 127 (2004) 127.

[57] G. De Moor, C. Bas, N. Charvin, E. Moukheiber, F. Niepceron, N. Breilly, J. André, E. Rossinot, E. Claude, N.D. Albérola, L. Flandin, Fuel Cells 12 (2012) 356.

[58] A.P. Young, J. Stumper, S. Knights, E. Gyenge, J. Electrochem. Soc. 157 (2010) B425.

[59] C. Lim, L. Ghassemzadeh, F. Van Hove, M. Lauritzen, J. Kolodziej, G.G. Wang, S. Holdcroft, E. Kjeang, J. Power Sources 257 (2014) 102.

[60] S. Zhang, X.Z. Yuan, R. Hiesgen, K.A. Friedrich, H. Wang, M. Schulze, A. Haug, H. Li, J. Power Sources 205 (2012) 290.

[61] S. Vengatesan, K. Panha, M.W. Fowler, X.Z. Yuan, H. Wang, J. Power Sources 207 (2012) 101.

[62] S. Kundu, M.W. Fowler, L.C. Simon, R. Abouatallah, N. Beydokhti, J. Power Sources 195 (2010) 7323.

[63] X. Huang, R. Solasi, Y. Zou, M. Feshler, K. Reifsnider, D. Condit, S. Burlatsky, T. Madden, J. Polym. Sci. Part B Polym. Phys. 44 (2006) 2346.

[64] N. Macauley, A.S. Alavijeh, M. Watson, J. Kolodziej, M. Lauritzen, S. Knights, G. Wang, E. Kjeang, J. Electrochem. Soc. 162 (2014) F98.

[65] CRC Handbook of Chemistry and Physics, eighty fifth ed., CRC Press, 2004.

[66] N. Macauley, L. Ghassemzadeh, C. Lim, M. Watson, J. Kolodziej, M. Lauritzen, S. Holdcroft, E. Kjeang, ECS Electrochem. Lett. 2 (2013) F33. 